\title{
Effect of Near-Side on-Line Bus Stop on Signalized Intersection Capacity
}

\author{
Jiao Yao, Jieqiong Xu and Jing Zhao \\ Business school, University of Shanghai for Science and Technology, Shanghai, \\ 200093, China \\ yaojiao@126.com
}

\begin{abstract}
In order to estimate the capacity of signalized intersection more exactly, this paper improves the bus blockage adjustment factor calculating model of HCM2010. The research is mainly focused on the near-side on-line bus stop. Based on the traffic flow theory and probability theory, the signalized intersection capacity is analyzed qualitatively and quantitatively. Three kinds of land-use of the bus loading lane were considerate. Then the study on the factors and its sensitivity analysis about the impact on the capacity due to the bus stop has been conducted. The results show that both the distance between the bus stop and intersection stop line and the arrival rate of the bus loading lane affect the signalized intersection capacity greatly. The distance between the bus stop and intersection stop line should be long enough to accommodate the queue length of the vehicles arrived during red time.
\end{abstract}

Keywords: traffic engineerin, bus blockage adjustment, probability theory, capacity, signalized intersection

\section{Introduction}

The setting of bus stops is the basic issue in bus system design, it has been further discussed in the layout of the bus stops[1-2], spacing of bus stops[3-4], locations[5-6] and forms[7-9] of bus stops in the past studies. The setting of bus stops can be classified into three types by their location: upstream and downstream of the intersection and section; by the form of the stops, they can be classified into near-side on-line and bus bay.

For the convenience of the flow distribution, the best location to set the bus stop is near the intersection. However, this location, especially the near-side on-line bus stop, has negative effect on the capacity of signalized intersection. The degree of the effects mainly depends on the geometry of sections, traffic flow and signal control.

Based on the intersection capacity calculating model of HCM2010[10], this study mainly focuses on the near-side on-line bus stop, improves the bus blockage adjustment factor calculating model.

\section{Literature Review}

The capacity of the intersection can be affected because the bus stop and block the subsequent vehicles, which shows in the rising of the delay and the decline of the capacity.

In the analysis of delay, Yagar[11] analyzed the effects of transit stops at signalized intersections on traffic delay qualitatively, under the different circumstances of intersection signal control strategies and bus arrival time. Besides, scholars like Wong[12], Ge Hongwei[13], Liao Chang[14] also gave the calculating models of influence on traffic delay. 
For the research of traffic capacity, the impact of passengers getting on and off in the bus stops within the range of $75 \mathrm{~m}$ from the intersection stop line is given in HCM2010[10]. But this model concerns only two factors, the number of lanes and the number of parking buses. Considering this, WANG Qian [15], Fetter[16]and YU Zongguang[17] added the factors such as the arrival rate of the bus, the length of time the bus stops, the distance between the stops and the intersections, the signal timing and so on. Besides, YANG Xiuying[18], YUAN Jing[19] studied the impact of bus stops on the traffic capacity of intersection entrance lanes, under the conditions of the bus lanes.

The studies above on the impact of near-side on-line bus stops on the capacity, assumed that the bus stops were located in the shared lane for through and right turn, there was no need to change the lane to pass through the intersection after the passengers on and off. However, in engineering practice, the lane that bus stops located can be set as the lane shared by through and right turn, exclusive right-turn lane and the lane shared by right turn and bus.

This paper will aim at the three conditions above, and modified models will be established for signal control intersection traffic capacity, and the sensitivity analysis is conducted on the basis of these models.

\section{Qualitative Analysis}

In this paper, the traffic capacity of the intersection under the effect of near-side on-line bus stop includes three cases: the bus stop locates in the lane shared by through and right turn, in exclusive right-turn lane and in lane shared by right turn and bus.

If the near side lane is the lane shared by through and right turn, the stops of bus in the lane will cause the following vehicles wait in line, the factors affect the traffic capacity of intersection entrance lanes are the duration that bus stops and the bus arrival time. When the bus arrives at the stop during the red time, all or part of the duration can be used for arrival, causing ignorable effects on the capacity of entrance approach; when the bus arrives at the stop during green time, part of the green duration need to be occupied for arrival, it will cause considerable effects on the capacity of entrance approach.

If the near-side lane is exclusive right-turn lane, considering the right turn lane is not affected by signal control, the bus cannot use the lane to stop during the red time, thus the capacity of the near-side line will be effected when the bus stops or changes lanes.

If the near side lane is the lane shared by right turn and bus, there will be no need for bus passing through the intersection to change lanes after stopping, just drive along the lane to the stop line. However, due to the signal control in through lanes, the bus may hinder the right turning traffic, that's to say, the effect on of traffic capacity is related to the time when the bus arrives at the stop.

\section{Model Foundation}

The effect of bus stop on signalized intersection capacity, essentially, is because that once the bus stops, the following vehicles of entrance approach will be hindered, resulting in the green loss time.

The adjustment factors for the capacity of the lanes which the near-side on-line bus stops located in are given as follows:

$$
\begin{aligned}
& f_{b b}=1-\frac{T_{b b}}{3600 N} \\
& T_{b b}=T_{g}+T_{c}+T_{d}
\end{aligned}
$$

where:

$f_{b b}=$ adjustment factor of the arrival rate of the bus loading lane; 
$T_{b b}=$ green loss time caused by the arrival of bus per hour, s;

$N=$ number of lanes;

$T_{g}=$ green loss time caused by the arrival of bus per hour, $\mathrm{s}$;

$T_{c}=$ green loss time caused by bus changing lanes per hour(For the lane the bus stop located in is exclusive right-turn lane), $\mathrm{s}$;

$T_{d}=$ green loss time caused by signal control of bus per hour (For the lane the bus stop located in is for through and right turn), s.

\subsection{The Lane the Bus Stop Located in is for Through and Right Turn}

\subsubsection{Model Hypothesis}

(1)Overtaking is forbidden in lanes, and vehicles of other lanes can't enter;

(2)All the vehicles have to stop in the duration of red time;

(3)The arrival of Bus follows uniform distribution.

\subsubsection{Model Establishment}

As the bus stop located in the lane for both through and right turn, after passengers getting on and off the bus, the bus can pass through the intersection directly without changing lanes, that makes the loss time is mainly the green loss time caused by the arrival of bus at bus stop.

Assume the distance from the bus stop to the stop line is $L_{b b}(\mathrm{~m})$, space headway of vehicles in front of stop line is $h_{d}(\mathrm{~m})$, thus the vehicle number in the range of $L_{b b}$ is $L_{b b} / h_{d}$. The impact of a single bus's arrival on the green loss time of the lane is related to bus arrival time $t_{b}\left(\mathrm{~s}\right.$, the moment the red light begins is 0 point) and time $T_{b s}(\mathrm{~s})$ required to reach $L_{b b} / h_{d}$ cars in the early time of red light. There are four situations as follows:

Situation 1: when $t_{b}<T_{b s}$ and $t_{b}<r-t_{s},\left(r\right.$ represents the red time of the lanes, s; $t_{s}$ represents the average bus stopping time in bus stop, s), bus can enter the bus stop smoothly and use the red time to complete the passengers' on and off service. In this situation, the green loss time $t_{g}$ caused by a single bus's arrival equals to 0 .

Situation 2: when $r-t_{s}<t_{b}<T_{b s}$, bus can also enter the bus stop smoothly, but use the red time to complete just part of the passengers' on and off service, bus will still be in the bus stop when the green time begins, makes the green loss time $t_{g}=t_{b}+t_{s}-r_{\circ}$

Situation 3: If bus arrives in red time, and $t_{b}>T_{b s}$, queue length will extend to the bus stop, bus will be not able to drive into bus stop and complete the passengers' on and off service until the green time begins. If bus arrives in green time, its arrival will lead to green loss time directly; therefore, green loss time $t_{g}=t_{s}$ 。

Situation 4: If bus arrives at the end of green time, and the passengers' on and off service can't be completed during green time, bus will have to wait till the next green time begins to pass through the intersection. In this situation, green loss time will always be $t_{g}$ $=C-t_{b}$ 。

Synthesis of the above four situations leads to the green loss time caused by the arrival of single bus:

$$
\begin{gathered}
T_{g t}=\left\{\begin{array}{cc}
0, & 0 \leq t_{b} \leq \mathrm{min}\left(T_{b s}, r-t_{s}\right) \\
t_{b}+t_{s}-r, & \min \left(T_{b s}, r-t_{s}\right)<t_{b} \leq \mathrm{min}\left(T_{b s}, r\right) \\
t_{s}, & \min \left(T_{b s}, r\right)<t_{b} \leq C-t_{s} \\
C-t_{b}, & C-t_{s}<t_{b} \leq C
\end{array}\right. \\
T_{b s}=\frac{L_{b b}}{q h_{d}}
\end{gathered}
$$


where:

$T_{g t}=$ green loss time caused by the stop of single bus per hour, s;

$q=$ the arrival rate of vehicles in lanes for both through and right turn, veh/s.

In the time of $[0 \mathrm{C}]$, the arrival of bus follows uniform distribution, its density function is shown in equation (5), and the green loss time caused by the arrival of bus is given in equation (6).

$$
\begin{gathered}
f\left(t_{b}\right)=\frac{1}{C} \\
T_{b b}=T_{g}=n_{b} \times E\left(t_{g t}\right)=\frac{n_{b}}{C} \int_{0}^{c} T_{g t} \cdot d t_{b} \\
=\left\{\begin{array}{cc}
\frac{n_{b} t_{s}\left(C-1 / 2 t_{s}-T_{b s}\right)}{C} & 0 \leq T_{b s} \leq r-t_{s} \\
\frac{n_{b}\left[\left(r-t_{0}\right)^{2}+(C-r) t_{s}\right]}{2 C} & r-t_{s}<T_{b s} \leq r \\
\frac{n_{b} t_{s}(C-r)}{C} & T_{b s} \geq r
\end{array}\right.
\end{gathered}
$$

Where:

$n_{b}=$ number of bus stopped in the bus stop located lane, veh。

\subsection{The Lane the Bus Stop Located in is Exclusive Right-Turn Lane}

\subsubsection{Model Hypothesis}

(1)Overtaking is forbidden in lanes, and vehicles of other lanes can't enter;

(2)The bus passing through the intersection must change lanes into the adjacent through lane once drive out the stop

(3)The arrival of Bus follows uniform distribution.

\subsubsection{Model Establishment}

As the bus stop located in the exclusive right-turn lane, green loss time caused by the arrival of bus contains green loss time caused by the arrival of bus and green loss time caused by bus changing lanes per hour.

The whole process of the stop of a bus will make the green time of right turn lane loss, the equation is given in equation(7), and the green loss time caused by all the buses is shown in equation(8).

$$
\begin{gathered}
T_{g t}=t_{s} \\
T_{g}=n_{b} \times E\left(t_{g t}\right)=n_{b} \times t_{s}
\end{gathered}
$$

Green loss time caused by single bus changing lanes $T_{c}$ is related to the moment the bus changing lanes $\left(t_{b}+t_{s}\right)$ and the time $T_{b s}$ the queue of vehicles in adjacent through lane extends out the bus stop. The two situations are as follows:

Situation 1: When it is ready to change lanes after driving out of bus stop, bus can enter into the adjacent lane without waiting. If the adjacent lane is green, or it's red but the vehicle is not lined up at the exit of the bus stop, in this situation, green loss time due to single bus changing lanes is 0 . 
Situation 2: When the bus is ready to change lanes after driving out of bus stop, if the adjacent lane is red, and the vehicle is lined up at the exit of the bus stop, traveling through bus must wait for the green time to enter into the adjacent lane.

Synthesis of the above two situations, green loss time caused by single bus changing lanes can be calculated by equation (9-11).

When $r \leq T_{b s}$ :

$$
T_{c t}=0
$$

Where:

$T_{c t}=$ green loss time of single bus caused by changing lanes per hour, $\mathrm{s}$.

When $r>T_{b s}$ and $t_{s} \leq T_{b s}$ :

$$
T_{c t}=\left\{\begin{array}{cc}
0 & t_{s} \leq t_{b}+t_{s} \leq T_{b s} \\
r-\left(t_{b}+t_{s}\right) & T_{b s}<t_{b}+t_{s} \leq r \\
0 & r<t_{b}+t_{s} \leq C+t_{s}
\end{array}\right.
$$

When $r>T_{b s}$ and $T_{b s}<t_{s}<r$ :

$$
T_{c t}=\left\{\begin{array}{cc}
r-\left(t_{b}+t_{s}\right) & t_{s} \leq t_{b}+t_{s} \leq r \\
0 & r<t_{b}+t_{s} \leq C+T_{b s} \\
C+r-\left(t_{b}+t_{s}\right) & C+T_{b s}<t_{b}+t_{s} \leq C+t_{s}
\end{array}\right.
$$

And green loss time caused by buses changing lanes is given in equation (12).

$$
T_{c}=n_{b} \times P_{T B} \times E\left(t_{c t}\right)=\frac{n_{b} \times P_{T B}}{C} \times \int_{0}^{c} t_{c t} d\left(t_{b}\right)
$$

where: in.

$P_{T B}=$ the proportion of bus driving through intersection in all the bus that need to stop

Simultaneous equation of (8) and (10) shows that, if the lane the bus stop located in exclusive right-turn lane, green loss time caused by the arrival of bus per hour is in equation(13).

$$
T_{b b}=\left\{\begin{array}{cl}
n_{b} t_{s} & r \leq T_{b s} \\
\min \left(\frac{n_{b} P_{T B}\left(r-t_{0}\right)^{2}}{2 C}+n_{b} t_{s},\left(r-t_{0}\right) \frac{3600}{C}+n_{b} t_{s}\right) & r>T_{b s}
\end{array}\right.
$$

\subsection{The Lane the Bus Stop Located in is for Right turn and Bus}

\subsubsection{Third-order Headings Model Hypothesis:}

(1)Overtaking is forbidden in lanes, and vehicles of other lanes can't enter;

(2)The bus passing through the intersection drive along its lane to the stop line after driving out of bus stop, and it will be under signal control;

(3)The arrival of Bus follows uniform distribution.

\subsubsection{Model Establishment:}

As the bus stop located in the lane for right turn and bus, green loss time caused by the arrival of bus contains green loss time caused by the arrival of bus and green loss time caused by signal control of bus per hour. 
The green loss time caused by the arrival of bus per hour is same as section 4.2 , the whole process of the stop of a bus will make the green time of right turn lane loss, the green loss time caused by all the buses is shown in equation(8).

Green loss time of bus caused by signal control $T_{d}$, is affected by the arrival time of bus at intersection. There are two situations as follows:

Situation 1: When the bus drive to the stop line after driving out of bus stop, and the through entrance lane is green, green loss time of bus driving through intersection caused by signal control is 0 .

Situation 2: When the bus drive to the stop line after driving out of bus stop, and the through entrance approach is red, the bus traveling through will have to wait for the green time to leave.

Synthesis of the above two situations, green loss time caused by signal control can be calculated as equation (14-15).

When $t_{s}+L_{b b} / v \leq r$ :

$$
T_{d t}=\left\{\begin{array}{cc}
r-\left(t_{b}+t_{s}+\frac{L_{b b}}{v}\right), & t_{s}+\frac{L_{b b}}{v} \leq t_{b}+t_{s}+\frac{L_{b b}}{v} \leq r \\
0, & r<t_{b}+t_{s}+\frac{L_{b b}}{v} \leq C \\
C+r-\left(t_{b}+t_{s}+\frac{L_{b b}}{v}\right), & C<t_{b}+t_{s}+\frac{L_{b b}}{v} \leq C+t_{s}+\frac{L_{b b}}{v}
\end{array}\right.
$$

Where:

$T_{d t}=$ green loss time of single bus caused by signal control per hour, $\mathrm{s}$; $V=$ speed of vehicles traveling through intersection, $\mathrm{m} / \mathrm{s}$.

When $t_{s}+L_{b b} / v>r$ :

$$
T_{d t}=\left\{\begin{array}{cc}
0 & t_{s}+\frac{L_{b b}}{V} \leq t_{b}+t_{s}+\frac{L_{b b}}{V} \leq C \\
C+r-\left(t_{b}+t_{s}+\frac{L_{b b}}{V}\right) & C<t_{b}+t_{s}+\frac{L_{b b}}{V} \leq C+r \\
0 & C+r<t_{b}+t_{s}+\frac{L_{b b}}{V} \leq C+t_{s}+\frac{L_{b b}}{V}
\end{array}\right.
$$

green loss time caused by the bus changing lanes is in equation (16).

$$
T_{d}=n_{b} \times P_{T B} \times E\left(t_{d}\right)=\frac{n_{b} \times P_{T B}}{C} \times \int_{0}^{c} t_{d} d\left(t_{b}\right)
$$

Simultaneous equation of (8) and (16) shows that, if the lane the bus stop located in is for right turn and bus, green loss time caused by the arrival of bus is in equation(17).

$$
T_{b b}=\min \left(\frac{n_{b} P_{T B} r^{2}}{2 C}+n_{b} t_{s}, \frac{3600 r}{C}+n_{b} t_{s}\right)
$$

\section{Model Analysis}

\subsection{The Lane the Bus Stop Located in is for through and Right turn}

In this section, the relationship between the effect degree of bus stop on capacity of signalized intersection, distance between stops and entrance approach $L_{b b}$, arrival rate $q$ of vehicles in the lane bus stop located and the time $T_{b s}$ the queue of vehicles in adjacent 
through lane extends out the bus stop will be analyzed. In this analysis, take the lane the bus stop located as the research object, take 1 as the number of lanes $\mathrm{N}$, take $120 \mathrm{~s}$ as signal cycle length $C$, take $80 \mathrm{~s}$ as red time length of lanes $r$, take $25 \mathrm{~s}$ as average bus stopping time $t_{s}$, take $60 \mathrm{veh} / \mathrm{h}$ as the number of bus stops at the bus stop per hour $n_{b}$, take $550 \mathrm{veh} / \mathrm{h}$ as arrival rate in lane shared by through and red turn vehicles $q$, take $50 \mathrm{~m}$ as distance between stop and entrance approaches $L_{b b}$, take $7.5 \mathrm{~m}$ as space headway of vehicles in front of stop line $h_{d}$.

Figure 1 shows the relationship between $L_{b b}$ and $\boldsymbol{f}_{b b}$. When the value of $L_{b b}$ is small, the adjustment factor is linear growth with the increasing of the distance between bus stop and entrance approaches, when the value of $L_{b b}$ is big, the growth of adjustment factor is getting slower, finally when $L_{b b} \geq r q h_{d}$, the adjustment factor reaches a steady state.

Figure 2 shows the relationship between $q$ and $f_{b b}$. When the value of $q$ is small, the adjustment factor reaches its max value and stays steady in a certain concentration range, when $q>L_{b b} / r h_{d}$, with the increase of $q$, the adjustment factor decreases sharply.

Figure 3 shows the relationship $T_{b s}$ and $f_{b b}$. When the value of $T_{b s}$ is small, the adjustment factor is linear growth with the increasing of $T_{b s}$, when the value of $T_{b s}$ is big, the growth of adjustment factor is getting slower, finally when $T_{b s} \geq r$, the adjustment factor reaches a steady state.

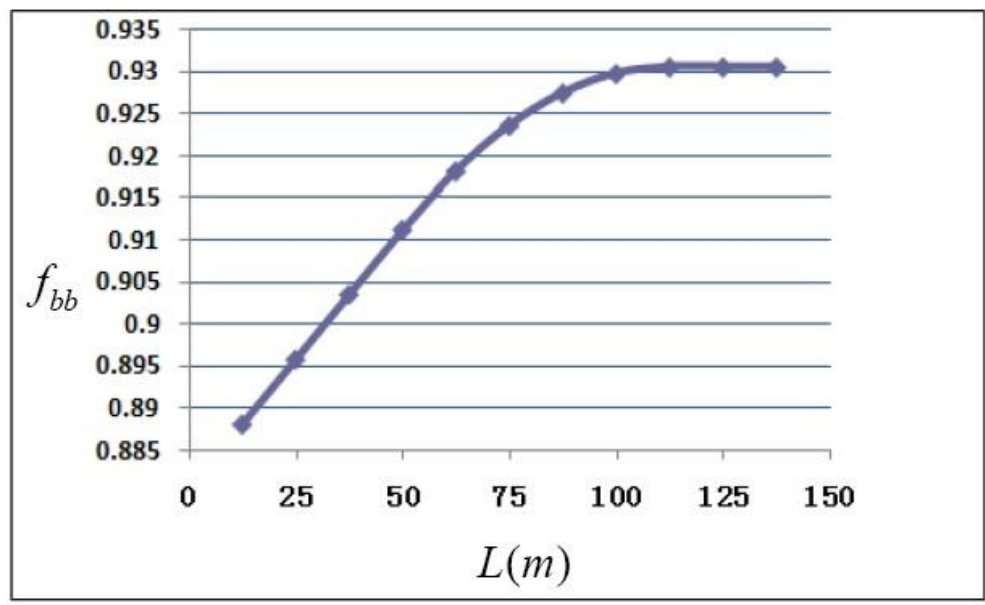

Figure 1. Relationship between $L_{b b}$ and $f_{b b}$

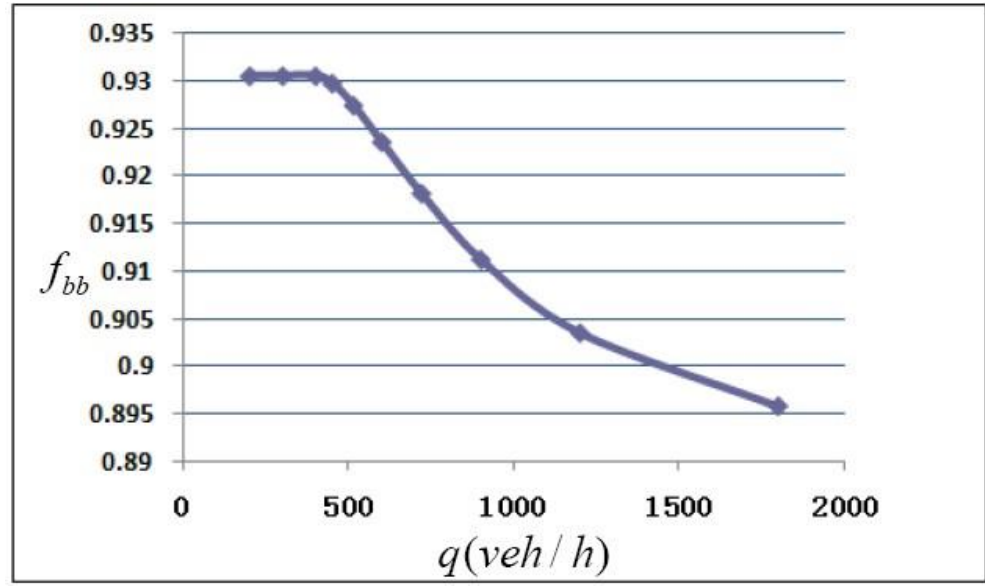

Figure 2. Relationship between $q$ and $f_{b b}$ 


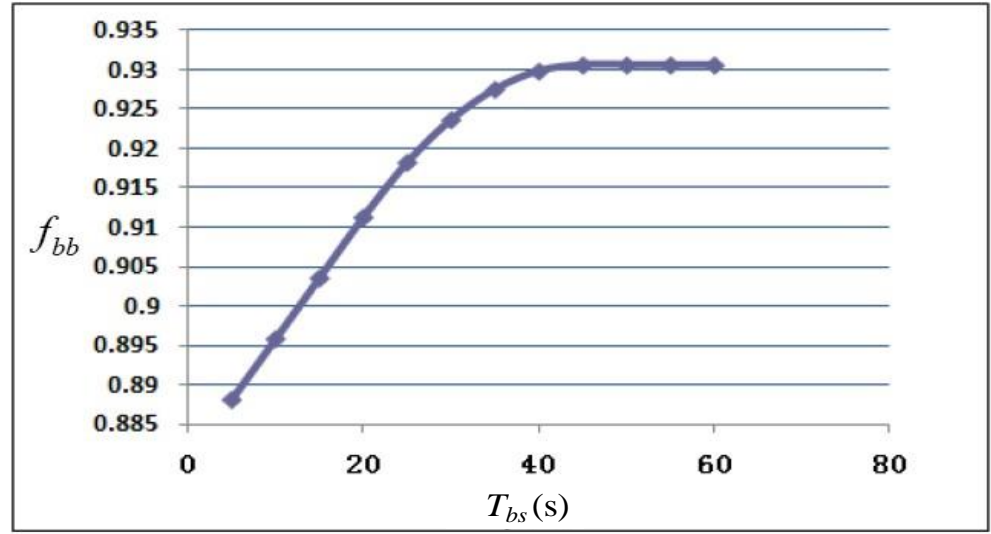

Figure 3. Relationship between $T_{b s}$ and $f_{b b}$

\subsection{The Lane the Bus Stop Located in is Exclusive right-turn lane}

According to section 4.2 and 4.3, there are two circumstances when the lane the bus stop located in is right-turn lane: the lane is exclusive right-turn lane, the bus has to change lanes to pass through intersection; the lane is for right turn and bus, the bus doesn't need to change lanes. Models of two circumstances has been established in preceding part of this paper, a comparative analysis will be discussed next, as shown in figure 4 below. Considering the fact that whether bus can change lanes or not depends on the arrival rate of adjacent through lanes, once the lane bus stop located in is exclusive right-turn lane, examples of two cases of $400 \mathrm{veh} / \mathrm{h}$ and $600 \mathrm{veh} / \mathrm{h}$ are taken in this section, other input parameter values are the same as the 5.1 section.

Figure 4 shows that when traffic flow of the adjacent through lane is low, if the lane bus stop located allows bus go through (lane for right turn and bus), its effect on capacity will be greater than if not; but when traffic flow of the adjacent through lane is high, if the lane bus stop located doesn't allow bus go through,(exclusive right-turn lane), its effect on capacity will be greater.

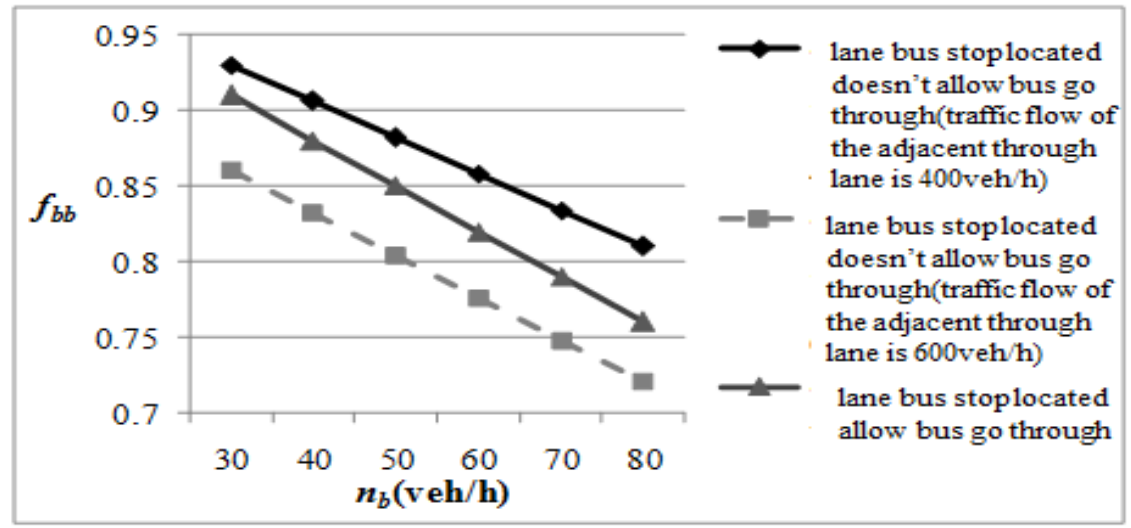

Figure 4. Relationship between nb and fbb

\section{Conclusion}

This paper established the calculating model of effect of near-side on-line bus stop on signalized intersection capacity. Three kinds of lane-use of the bus loading lane were considered. The following conclusions can be drawn by the sensitivity analysis of model parameters:

(1)The effect of near-side on-line bus stop on signalized intersection capacity is negatively correlated with the distance between bus stop and entrance approaches and the 
time the queue of vehicles extends out the bus stop, but positively correlated with the arrival rate of vehicles in the bus stop located lane.

(2)As for the situation that bus stop located in the lane for both through and right turn, bus stop will make subsequent vehicles wait in queue. When the distance between the bus stop and intersection stop line is long enough to accommodate the queue length of the vehicles arrived during red time, the bus stop has little effect on the capacity of entrance approaches.

(3)When traffic flow of the adjacent through lane is low, if the lane bus stop located in is the lane for right turn and bus, its effect on capacity is greater than in exclusive rightturn lane; but when traffic flow of the adjacent through lane is high, if the lane bus stop located in exclusive right-turn lane, its effect on capacity is greater.

\section{Acknowledgment}

This paper is a revised and expanded version of a paper entitled "study of signalized intersection affected by bus station at upstream" presented at COMCOMS 2015, Hanoi, Vietnam, October 22-24, 2015. This research was funded by the Chinese National Natural Science Fundamental Research Program (61403255); Shanghai Training Program of Youth Teacher in Universities (slg12009). Authors would like to thank all these support.

\section{References}

[1] W. Wei, Y. Xinmiao and C. Xuewu, "City Public transportation system planning and management" Beijing Science Press, (2002).

[2] P. Ming-bao, Z. Si-lin and L. Chun-xia, "Bi-level Programming of urban bus stop locating" Journal of Highway and Transportation Research and Development, vol. 30, no. 3, (2013).

[3] S.C. Wirasinghe and N. S. Ghoneim, "Spacing of bus stops for many to many travel demand Transportation Science", vol. 15, no. 3, (1981).

[4] A.A. Saka, "Model for determining optimum bus-stop spacing in urban areas." Journal of Transportation Engineering, vol. 127, no. 3 (2001).

[5] R. Rajbhandari, S. I. Chien and J. R. Daniel, "Estimation of bus dwell times with automatic passenger counter information" Transportation Research Record Annual Meeting, Washington D.C., USA, (2003).

[6] W. Zhizhou, M. Wanjing and Y, Xiaoguang, "Optimal location of adjacent bus stops on condition of bus signal priority" Journal of Highway and Transportation Research and Development, vol. 25, no. 01, (2008).

[7] P. Chunlu and P. Guoxiong, "Discussion about a new method of bus stop on arterial street" China Civil Engineering Journal, vol. 36, no. 1, (2003).

[8] Y. Xiaoguang, Z. Hailei and W. Tao, "Research on transverse-arranged bus bay" Journal of Highway and Transportation Research and Development, vol. 24, no. 9, (2007).

[9] Y. Xiao-guang, X. Hui and G. Xin. "Study of setting conditions of bus bay stop of single lane" Journal of Highway and Transportation Research and Development, vol. 27, no. 12, (2010).

[10] "Transportation Research Board" Highway Capacity Manual 2010, Transportation Research Board, Washington, D.C. (2010).

[11] S. Yagar, M.A.P. Jacques, "Representing the effects of transit stops at signalized intersections" International Symposium on Highway Capacity, Balkema, Netherlands, (1991).

[12] S. C. Wong, H. Yang and W.S. A Yeung, "Delay at signal-controlled intersection with bus stop upstream" Journal of Transportation Engineering, vol. 124, no. 3 ,(1998).

[13] G. Hongwei, W. Wei and C. Xuewu, "Traffic delay at signal-control intersection with bus stop upstream” Journal of Southeast University (Natural Science Edition), vol. 36, no. 6, (2006).

[14] L. Chang, "Influence of bus stops on traffic delay at signalized intersection. Beijing: Beijing Jiaotong University, (2011).

[15] W. Qian and Y. Xiaoguang. "Impact of bus stop on delay and capacity of shared approaches at signalized intersections" China Civil Engineering Journal, vol. 36, no. 1, (2003).

[16] W.W. Fetter, "Effect of far-side and side-street bus stops on the saturation flow rate of signalized intersection" Carolina: North Carolina State University (2007).

[17] Y. Zongguang, "The capacity change considering the influence the bus stop" Chendu: Southweat Jiaotong University, (2009).

[18] Y. Xiuying, "The research on the impact of bus lane on signal intersection capacity" Shanghai: Tongji University, (2002).

[19] Y. Jing, "The influence analysis of bus stop on road capacity" Chendu: Southweat Jiaotong University, (2013). 


\section{Authors}

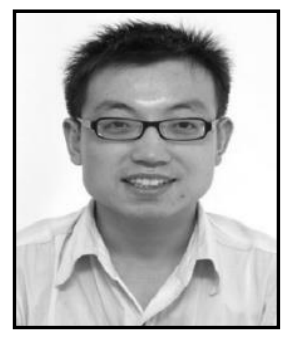

Jiao Yao, Assistant Professor in the Department of Transportation System Engineering, business school, University of Shanghai for Science and Technology, Shanghai, China. He received $\mathrm{PhD}$ in College of Transportation Engineering, Tongji University, Shanghai, China. His research interest is Intelligent Transportation Control, Connected Vehicle, and Traffic System Engineering.

Email: yzaojiao@126.com

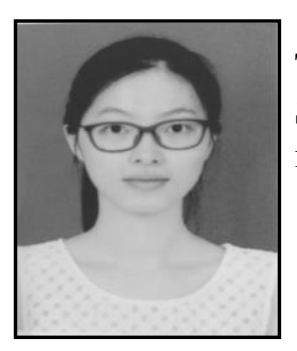

Jieqiong $\mathbf{X u}$, Master degree candidate in the Department of Transportation System Engineering, business school, University of Shanghai for Science and Technology, Shanghai, China. Her research interest is Intelligent Transportation Control.

Email:xu753xu@qq.com

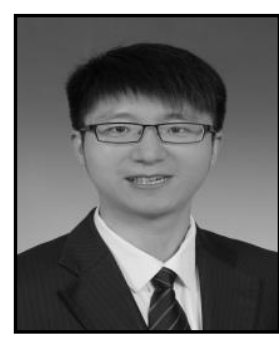

Jing Zhao, Assistant Professor in the Department of Transportation System Engineering, business school, University of Shanghai for Science and Technology, Shanghai, China. He received $\mathrm{PhD}$ in College of Transportation Engineering, Tongji University, Shanghai, China. His research interest is Traffic Control and Management.

Email: jing_zhao_traffic@163.com 